Journal of Algebraic Combinatorics, 21, 185-202, 2005 c) 2005 Springer Science + Business Media, Inc. Manufactured in The Netherlands.

\title{
Generalized Exponents and Forms
}

\author{
ANNE V. SHEPLER \\ Mathematics Department, University of North Texas, Denton, Texas, U.S.A.
}

ashepler@unt.edu

Received January 21, 2003; Revised February 10, 2004; Accepted April 8, 2004

\begin{abstract}
We consider generalized exponents of a finite reflection group acting on a real or complex vector space $V$. These integers are the degrees in which an irreducible representation of the group occurs in the coinvariant algebra. A basis for each isotypic component arises in a natural way from a basis of invariant generalized forms. We investigate twisted reflection representations ( $V$ tensor a linear character) using the theory of semi-invariant differential forms. Springer's theory of regular numbers gives a formula when the group is generated by $\operatorname{dim} V$ reflections. Although our arguments are case-free, we also include explicit data and give a method (using differential operators) for computing semi-invariants and basic derivations. The data give bases for certain isotypic components of the coinvariant algebra.
\end{abstract}

Keywords: reflection group, invariant theory, generalized exponents, Coxeter group, fake degree, hyperplane arrangement, derivations

\section{Introduction}

Real and complex finite reflection groups exhibit fascinating numerology. The exponents and coexponents of the group arise in numerous ways, for example, as the degrees of the reflection representation and its dual in the coinvariant algebra and also as the degrees of generating invariant differential forms and derivations. We investigate the numerology of twisted reflection representations here.

Let $V:=\mathbb{C}^{\ell}$ and recall that a reflection is an element of $G L(V)$ whose fixed point set is a hyperplane in $V$. Let $G$ be a reflection group, i.e., a finite subgroup of $G L(V)$ generated by reflections. Such groups are often called pseudo-reflection groups and include the Weyl and Coxeter groups. (See Orlik and Terao [12], Kane [5], or Smith [15] for basic notions.) We assume all $G$-modules are $\mathbb{C} G$-modules. For any $G$-module $U$ and irreducible $G$-module $M$, let $U^{M}$ be the isotypic component of $U$ of type $M$, i.e., the direct sum of those $G$ submodules of $U$ isomorphic to $M$. Let $U^{G}:=\{u \in U: g u=u$ for all $g \in G\}$ denote the set of $G$-invariants. For any linear character $\chi: G \rightarrow \mathbb{C}^{*}$, let $\mathbb{C}_{\chi}$ be a one-dimensional $G$-module affording $\chi$ and let $U^{\chi}:=U^{\mathbb{C}_{\chi}}=\{u \in U: g u=\chi(g) u$ for all $g \in G\}$ be the set of $\chi$-invariants in $U$. The reflection group $G$ acts contragradiently on $V^{*}$ and thus on the symmetric algebra $S:=S\left(V^{*}\right)$, which we identify with the algebra of polynomial functions on $V$. The algebra $S$ is naturally graded by polynomial degree. Let $I \subset S$ be the Hilbert ideal generated by the invariant polynomials of positive degree. Chevalley [4] and Shephard and Todd [13] show that $S^{G}=\mathbb{C}\left[f_{1}, \ldots, f_{\ell}\right]$ for some homogeneous polynomials $f_{1}, \ldots, f_{\ell}$ 
called basic invariants. The algebra $S / I$ is called the coinvariant algebra. Chevalley also proved that $S / I$ is isomorphic to the regular representation and that $S \simeq S^{G} \otimes S / I$ as $G$-modules.

The coinvariant algebra $S / I$ inherits the grading on $S$. For any irreducible $G$-module $M$, the isotypic component $(S / I)^{M}$ decomposes as $M_{1} \oplus M_{2} \oplus \cdots \oplus M_{\operatorname{dim} M}$ for some homogeneous subspaces $M_{i} \simeq M$ of degree $e_{i}(M)$. We call $e_{1}(M), e_{2}(M), \ldots, e_{\operatorname{dim} M}(M)$ the M-exponents. For any linear character $\chi$ of $G$, the $\chi$-exponent is the $\mathbb{C}_{\chi}$-exponent, denoted $e(\chi)$. Let $m_{1}, \ldots, m_{\ell}$ be the $V$-exponents, called the exponents of the group, and assume that $m_{1} \leq \cdots \leq m_{\ell}$. Similarly, let $m_{1}^{*}, \ldots, m_{\ell}^{*}$ be the $V^{*}$-exponents, called the coexponents of the group, and assume that $m_{1}^{*} \geq \cdots \geq m_{\ell}^{*}$. The exponents and coexponents of the group indicate the invariant theory of differential forms and derivations (see Section 3). The coexponents also express the cohomology of the complement of the hyperplane arrangement (see Orlik and Solomon [12, Cor. 6.62]).

Springer [18] studies generalized exponents, and Stembridge [22] gives a combinatorial interpretation for the infinite family $G(r, p, \ell)$ and other wreath products. The associated generating function is called the "fake degree" (see Broué, Malle, Michel [3], for example). Ariki et al. [1] give a basis for the coinvariant algebra for the monomial groups $G(r, 1, \ell)$ consisting of higher Specht polynomials associated to Young diagrams. Morita and Yamada [9] develop a theory of higher Specht polynomials for the groups $G(r, p, \ell)$. The exceptional reflection groups do not lend themselves to the same kind of combinatorial analysis.

We relate the isotypic component $(S / I)^{M}$ with the space $S \otimes M^{*}$. The reflection group $G$ acts naturally on $S \otimes M^{*}$ and the rank of $\left(S \otimes M^{*}\right)^{G}$ as an $S^{G}$-module is $\operatorname{dim}_{\mathbb{C}} M$ (see [17, Lemma 2]). The module $S \otimes M^{*}$ also inherits a grading from $S$ : let $q_{1}, \ldots, q_{r}$ be a fixed basis of $M^{*}$ and suppose $\omega=\sum_{i} w_{i} \otimes q_{i} \in S \otimes M^{*}$; if the polynomial coefficients $w_{i}$ are all homogeneous of degree $p$ in $S$, then we say that $\omega$ is homogeneous of polynomial degree $p$.

In Section 2, we remark that the polynomial coefficients of any $S^{G}$-basis of $\left(S \otimes M^{*}\right)^{G}$ form a linear basis of the isotypic component $(S / I)^{M}$. Thus, the $M$-exponents are just the degrees of a homogeneous basis of $\left(S \otimes M^{*}\right)^{G}$ over $S^{G}$. We begin our investigation of twisted reflection representations in Section 3 with some background and results on semi-invariant differential forms. In Section 4, we use information about semi-invariant polynomials, forms, and derivations to describe generalized exponents for $\chi V:=V \otimes \mathbb{C}_{\chi}$, where $\chi$ is a linear character of $G$. The main result of this section is Corollary 13 relating $\chi$, $\bar{\chi}, \chi V$, and $\chi V^{*}$-exponents. We apply Springer's Theory of regular numbers in Section 4 to reflection groups generated by $\operatorname{dim} V$ reflections. In Section 5, we discuss a method for computing derivations and semi-invariants. Computational results are given in tables at the end, although previous results are obtained case-free. We include the explicit $\chi V$ exponents for all of the linear characters $\chi$ and exceptional irreducible reflection groups. Previous research has centered on Coxeter groups and the infinite family $G(r, p, \ell)$. We hope the approach here will be helpful in understanding the coinvariant algebra of exceptional reflection groups. 


\section{Bases for isotypic components of the coinvariant algebra}

Suppose $M$ is an irreducible representation of the reflection group $G$. Solomon [17, Lemma 2] shows that the $M$-exponents are the degrees of a homogeneous basis of $\left(S \otimes M^{*}\right)^{G}$ over $S^{G}$. We point out a slightly stronger result:

Proposition 1 Let $M$ be an irreducible G-module. Then a natural G-isomorphism

$$
M \otimes\left(S \otimes M^{*}\right)^{G} \simeq S^{G} \otimes(S / I)^{M}
$$

provides an injective map

$$
S^{G} \text {-bases of }\left(S \otimes M^{*}\right)^{G} \rightarrow \mathbb{C} \text {-bases of }(S / I)^{M}:
$$

The polynomial coefficients of an $S^{G}$-basis of $\left(S \otimes M^{*}\right)^{G}$ form a $\mathbb{C}$-basis of $(S / I)^{M}$ modulo I. Hence, the $M$-exponents are the degrees of a homogeneous basis of $\left(S \otimes M^{*}\right)^{G}$ over $S^{G}$.

Proof: Note that $\left(S \otimes M^{*}\right)^{G} \simeq\left(S^{G} \otimes S / I \otimes M^{*}\right)^{G} \simeq S^{G} \otimes\left(S / I \otimes M^{*}\right)^{G}$ by Chevalley's Theorem and $\left(S / I \otimes M^{*}\right)^{G} \simeq \operatorname{Hom}_{G}(M, S / I) \simeq \operatorname{Hom}_{G}\left(M,(S / I)^{M}\right)$. But $M \otimes \operatorname{Hom}_{G}\left(M,(S / I)^{M}\right) \simeq(S / I)^{M}$ as $M$ is irreducible. Suppose $\omega_{1}, \ldots, \omega_{r}$ form an $S^{G}$-basis for $\left(S \otimes M^{*}\right)^{G}$ and write each $\omega_{k}$ as $\omega_{k}=\sum_{j=1, \ldots r} s_{j k} \otimes m_{j}$ for some fixed basis $m_{1}, \ldots, m_{r}$ of $M^{*}$. Then under a composition

$$
M \otimes\left(S \otimes M^{*}\right)^{G} \simeq S^{G} \otimes(S / I)^{M} \rightarrow(S / I)^{M}
$$

(where $a \otimes(b+I) \mapsto a b+I)$,

$$
\left\{m_{j} \otimes \omega_{k}: 1 \leq k, j \leq r\right\} \mapsto\left\{s_{j k}+I: 1 \leq k, j \leq r\right\}
$$

One may verify that this last set spans $(S / I)^{M}$ over $\mathbb{C}$ and thus forms a basis.

\section{Twisted reflection representations and differential forms}

We consider twisted reflection representations of the group $G$ and relate components of the coinvariant algebra to differential forms. Identify $\Omega^{p}:=S \otimes \wedge^{p} V^{*}$ with the space of differential $p$-forms on $V$ and set $\Omega:=\bigoplus_{p=0}^{\ell} \Omega^{p}$. Let $d: \Omega^{p} \rightarrow \Omega^{p+1}$ be the usual exterior derivative and let vol be the volume form on $V$ (defined up to a nonzero scalar). Note that $d x=1 \otimes x$ under the identification $\Omega^{0}=S$ for any $x$ in $V^{*}$.

Semi-invariant differential forms are related to certain isotypic components of the coinvariant algebra. Consider a linear character of the reflection group, $\chi: G \rightarrow \mathbb{C}^{*}$. We call $\chi V:=V \otimes \mathbb{C}_{\chi}\left(\right.$ or $\left.\chi V^{*}:=V^{*} \otimes \mathbb{C}_{\chi}\right)$ a twisted reflection representation. If $G$ is irreducible, the last proposition implies that an $S^{G}$-basis of $\left(\Omega^{1}\right)^{\chi} \simeq\left(S \otimes V^{*} \otimes \mathbb{C}_{\bar{\chi}}\right)^{G}$ yields a linear basis for the isotypic component of the coinvariant algebra whose type is $\chi V$. 
We recall some facts about invariant differential forms and derivations. Let $f_{1}, \ldots, f_{\ell}$ be a set of basic invariants. The exterior derivative $d$ commutes with the group action on $\Omega$ and $d f_{1}, \ldots, d f_{\ell}$ are invariant 1-forms. These forms generate $\left(S \otimes V^{*}\right)^{G}$ as a free $S^{G}$-module. The exponents of the group are thus the integers $m_{1}=\operatorname{deg} f_{1}-1, \ldots, m_{\ell}=\operatorname{deg} f_{\ell}-1$. We regard $S \otimes V$ as the $S$-module of derivations (or vector fields) on $V$. Generators of $(S \otimes V)^{G}$ over $S^{G}$ are called basic derivations (see [12, Def. 6.50]). The (polynomial) degrees of a set of homogeneous basic derivations are the coexponents of the group.

Solomon [16] shows that $d f_{1}, \ldots, d f_{\ell}$ generate the $S^{G}$-module of invariant different forms as an exterior algebra: for each $p$,

$$
\left(\Omega^{p}\right)^{G}=\bigoplus_{i_{1} \leq \cdots \leq i_{p}} S^{G} d f_{i_{1}} \wedge \cdots \wedge d f_{i_{p}}
$$

and thus $\Omega^{G}=\bigwedge_{S^{G}}\left(\Omega^{1}\right)^{G}$. We recall a related result for $\Omega^{\chi}$, the $S^{G}$-module of $\chi$-invariant forms. The space of $\chi$-invariant polynomials, $S^{\chi}$, has rank 1 as an $S^{G}$-module. Let $Q_{\chi} \in S$ be a (homogeneous) generator:

$$
S^{\chi}=Q_{\chi} S^{G}
$$

(Note that $Q_{\chi}$ is only defined up to a nonzero scalar. Also note that the degree of $Q_{\chi}$ is the $\chi$-exponent, $e_{\chi}$ (see Lemma 5).) The polynomial $Q_{\chi}$ divides the exterior product of any two $\chi$-invariant forms (see Shepler [14]) and we define a multiplication on $\Omega^{\chi}$ called $\chi$-wedging:

$$
\omega \curlywedge \eta:=\frac{\omega \wedge \eta}{Q_{\chi}}
$$

Define $\curlywedge_{S^{G}}^{p} M:=Q_{\chi}^{1-p} \bigwedge_{S^{G}}^{p} M$ for any $S^{G}$-module $M$ of $\chi$-invariant forms; then $\chi$-wedging endows $\curlywedge_{S^{G}} M:=\bigoplus_{p=0}^{\ell} \curlywedge_{S^{G}}^{p} M$ with the structure of an exterior algebra. Let det : $G \rightarrow \mathbb{C}^{*}$ be the determinant character of $G$ on $V$. We recall a criterion from Shepler [14] for a set of forms to generate $\Omega^{\chi}$ as an algebra:

Theorem 2 Let $\chi$ be a linear character of $G$ and let $\omega_{1}, \ldots, \omega_{\ell}$ be homogeneous $\chi$-invariant 1-forms. Then the following are equivalent:

1. Up to a nonzero scalar, $\omega_{1} \curlywedge \cdots \curlywedge \omega_{\ell}=Q_{\chi \text { det }}$ vol.

2. The forms $\omega_{i}$ generate $\Omega^{x}$ :

$$
\left(\Omega^{p}\right)^{\chi}=\bigoplus_{i_{1}<\cdots<i_{p}} S^{G} \omega_{i_{1}} \curlywedge \cdots \curlywedge \omega_{i_{p}} \quad \text { for } \quad p=1, \ldots, \ell
$$

Furthermore, there exist forms satisfying (1) and (2), and $\Omega^{\chi}$ is an exterior algebra:

$$
\Omega^{\chi}=\curlywedge_{S^{G}}\left(\Omega^{1}\right)^{\chi}
$$


We say that $\omega_{1}, \ldots, \omega_{\ell}$ generate $\Omega^{\chi}$ if they generate $\Omega^{\chi}$ as an $S^{G}$-module via $\chi$-wedging in the sense of Theorem 2. We assume such generators are homogeneous. Although the $\omega_{i}$ are not unique, their degrees are unique. Proposition 1 then implies

Corollary 3 Suppose that $G$ is irreducible and $\chi$ is a linear character of G. Let $\omega_{1}, \ldots, \omega_{\ell}$ generate $\Omega^{x}$ and write each $\omega_{k}$ as $\sum_{i=1}^{\ell} w_{i k} d x_{i}$, where the $x_{i}$ form a basis of $V^{*}$. Then

$$
\left\{w_{i k}+I: i, k=1, \ldots, \ell\right\}
$$

is a $\mathbb{C}$-basis for the isotypic component $(S / I)^{\chi V}$. The degrees of a generating set of $\Omega^{\chi}$ are the $\chi V$-exponents.

Corollary 4 Let $\chi$ be a linear character of G. Suppose generators of $\Omega^{x}$ have degrees $e_{1}, \ldots, e_{\ell}$. Then a (homogeneous) basis of the $S^{G}$-module $(S \otimes V)^{x \operatorname{det}}$ has degrees $\mathrm{deg}$ $Q_{\chi \operatorname{det}}+\operatorname{deg} Q_{\chi}-e_{i}$ for $i=1, \ldots, \ell$.

Proof: Let $\Upsilon^{p}:=S \otimes \bigwedge^{p} V$. The $G$-equivariant perfect pairing $\bigwedge^{p} V \otimes \bigwedge^{\ell-p} V \rightarrow \mathbb{C}_{\overline{\mathrm{det}}}$ gives a degree-preserving duality between semi-invariant differential forms and vector field forms:

$$
\left(\Upsilon^{p}\right)^{\chi \cdot \operatorname{det}} \simeq\left(\Omega^{\ell-p}\right)^{\chi}
$$

as $S^{G}$-modules. Hence, by Theorem $2,(S \otimes V)^{\chi \operatorname{det}}=\left(\Upsilon^{1}\right)^{\chi \operatorname{det}} \simeq\left(\Omega^{\ell-1}\right)^{\chi}=\curlywedge^{\ell-1}\left(\Omega^{1}\right)^{\chi}$. Theorem 2 also implies that $e_{1}+\ldots+e_{\ell}=(\ell-1) \operatorname{deg} Q_{\chi}+\operatorname{deg} Q_{\chi \text { det }}$. Hence, generators of $(S \otimes V)^{x \text { det }}$ have degrees

$$
(2-\ell) \operatorname{deg} Q_{\chi}+\left(e_{1}+\cdots+\hat{e}_{i}+\cdots+e_{\ell}\right)=\operatorname{deg} Q_{\chi}+\operatorname{deg} Q_{\chi \operatorname{det}}-e_{i}
$$

for $i=1, \ldots, \ell$.

\section{Exponents of twisted reflection representations}

We collect some observations about $\chi$-invariant forms, where $\chi$ is any linear character of the reflection group $G$. These observations in turn provide various combinatorial relations among $\chi$-exponents and $\chi V$-exponents. The main result of this section is Corollary 13 .

Let $\mathcal{A}$ denote the collection of reflecting hyperplanes in $V$ for the group $G$. For each hyperplane $H$ in $\mathcal{A}$, let $s_{H}$ be a reflection of maximal order fixing $H$ pointwise. Let $l_{H}$ in $V^{*}$ be a linear form with $H=\operatorname{ker} l_{H}$. Stanley [19] gives a formula for $Q_{\chi}$ :

$$
Q_{\chi}=\prod_{H \in \mathcal{A}} l_{H}^{a_{H}(\chi)},
$$


where $a_{H}(\chi)$ is the unique integer satisfying $0 \leq a_{H}(\chi)<\operatorname{order}\left(s_{H}\right)$ and $\chi\left(s_{H}\right)=$ $\operatorname{det}\left(s_{H}\right)^{-a_{H}(\chi)}$. Define

$$
Q:=Q_{\overline{\mathrm{det}}}=\prod_{H \in \mathcal{A}} l_{H},
$$

the polynomial which defines the hyperplane arrangement $\mathcal{A}$. Steinberg [20] gave a proof that the determinant of the Jacobian derivative of a set of basic invariants is $Q_{\text {det }}$ up to a nonzero scalar. The image of this Jacobian determinant is nonzero in the coinvariant algebra (for example, see [10, Lemma 6] or [15, Cor. 6.5.2]). Hence $e(\overline{\operatorname{det}})=\operatorname{deg} Q$, the number of reflecting hyperplanes. Similarly, $e(\operatorname{det})=\operatorname{deg} Q_{\text {det }}$, the number of reflections in $G$. In fact, since each $Q_{\chi}$ divides $Q_{\text {det }}$, we have the following well-known generalization:

Lemma 5 For any linear character $\chi$ of $G$, the image of the polynomial $Q_{\chi}$ is nonzero in the coinvariant algebra and the $\chi$-exponent is $e(\chi)=\operatorname{deg} Q_{\chi}$.

The next lemma follows directly from Stanley's formula. The lemma after gives generators of $\Omega^{\bar{\chi}}$ in terms of generators of $\Omega^{\chi}$. Corollary 8 is a result of Terao [23] (see [12,6.61]). Proposition 9 relates the $\chi V$-exponents to the exponents $m_{i}$ and the coexponents $m_{i}^{*}$ of the reflection group $G$.

Lemma 6 Let $\chi$ be a linear character of G. Up to a complex scalar,

$$
Q_{\chi} Q_{\bar{\chi} \cdot \operatorname{det}}=Q_{\text {det }}
$$

Lemma 7 Let $\chi$ be a linear character of $G$. Suppose $\omega_{1}, \ldots, \omega_{\ell}$ generate $\Omega^{\chi}$ and let $\eta_{i}:=\left(Q_{\bar{\chi}} / Q_{\chi}\right) \omega_{i}$. Then $\eta_{1}, \ldots, \eta_{\ell}$ generate $\Omega^{\bar{\chi}}$.

Proof: We first observe that $Q_{\chi}$ divides each $Q_{\bar{\chi}} \omega_{k}$. Choose $H$ in $\mathcal{A}$ with $a:=a_{H}(\chi) \neq$ 0 . Fix a basis $x_{1}, \ldots, x_{\ell}$ of $V^{*}$ so that $l_{H}=x_{1}$ and the matrix of the reflection $s_{H}$ is diagonal. Let $\omega$ be some generator $\omega_{k}=\sum_{i} w_{i} d x_{i}$. Since $\omega$ is invariant, $x_{1}^{a}$ divides $w_{i}$ whenever $i \neq 1$ and $x_{1}^{a-1}$ divides $w_{1}$. Stanley's formula for $Q_{\chi}$ (Equation 2) implies that $x_{1}$ divides $Q_{\bar{\chi}}$, and hence $l_{H}^{a}$ divides $Q_{\bar{\chi}} \omega$. As $H$ was arbitrary, $Q_{\chi}$ divides $Q_{\bar{\chi}} \omega$, and each $\eta_{i}$ is $\bar{\chi}$-invariant. By Lemma 6 and Theorem 2,

$$
\begin{aligned}
\eta_{1} \wedge \cdots \wedge \eta_{\ell} & =Q_{\bar{\chi}}^{\ell} Q_{\chi}^{-\ell} \omega_{1} \wedge \cdots \wedge \omega_{\ell}=Q_{\bar{\chi}}^{\ell} Q_{\chi}^{-\ell} Q_{\chi}^{\ell-1} Q_{\chi \cdot \operatorname{det}} \text { vol } \\
& =Q_{\bar{\chi}}^{\ell-1} Q_{\chi}^{-1} Q_{\text {det }} \text { vol }=Q_{\bar{\chi}}^{\ell-1} Q_{\bar{\chi} \cdot \operatorname{det} \text { vol }}
\end{aligned}
$$

up to a nonzero scalar. Hence, by Theorem $2, \eta_{1}, \ldots, \eta_{\ell}$ generate $\Omega^{\bar{x}}$.

Corollary 8 Generators of $\Omega^{\operatorname{det}}$ have degrees $\operatorname{deg} Q_{\mathrm{det}}-m_{i}^{*}$ for $1 \leq i \leq \ell$. Generators of $\Omega^{\overline{\mathrm{det}}}$ have degrees $\operatorname{deg} Q_{\overline{\mathrm{det}}}-m_{i}^{*}$ for $1 \leq i \leq \ell$. 
Proof: Apply Corollary 4 to the case $\chi=\operatorname{det}^{-1}=\overline{\operatorname{det}}$ and recall that invariant derivations have degrees $m_{1}^{*}, \ldots, m_{\ell}^{*}$. Lemma 7 then implies the first claim.

Proposition 9 Let $\chi$ be a linear character of $G$ and let $\omega_{1}, \ldots, \omega_{\ell}$ generate $\Omega^{\chi}$ with $\operatorname{deg} \omega_{i} \leq \operatorname{deg} \omega_{i+1}$. Then for each $i$,

$$
\operatorname{deg} Q_{\chi}-m_{i}^{*} \leq \operatorname{deg} \omega_{i} \leq \operatorname{deg} Q_{\chi}+m_{i}
$$

Proof: If $f_{1}, \ldots, f_{\ell}$ are basic invariants, then the forms $Q_{\chi} d f_{1}, \ldots, Q_{\chi} d f_{\ell}$ are independent over $S^{G}$, and hence $\operatorname{deg} \omega_{i} \leq \operatorname{deg} Q_{\chi}+m_{i}$ for each $i$. Let $\mu_{1}, \ldots, \mu_{\ell}$ be generators of $\Omega^{\text {det }}$ with $\operatorname{deg} \mu_{k}=\operatorname{deg} Q_{\text {det }}-m_{k}^{*}$ (using Corollary 8 ). Note that $Q_{\bar{\chi} \cdot \operatorname{det}} \omega_{1}, \ldots, Q_{\bar{\chi} \cdot \operatorname{det}} \omega_{\ell}$ are det-invariant forms independent over $S^{G}$, and hence (by Lemma 6)

$$
\operatorname{deg} Q_{\operatorname{det}}-m_{i}^{*}=\operatorname{deg} \mu_{i} \leq \operatorname{deg} Q_{\bar{\chi} \cdot \operatorname{det}} \omega_{i}=\operatorname{deg} Q_{\operatorname{det}}-\operatorname{deg} Q_{\chi}+\operatorname{deg} \omega_{i} .
$$

Proposition 10 Let $\chi \neq 1$ be a linear character of $G$. Suppose $\omega_{1}, \ldots, \omega_{\ell}$ generate $\Omega^{\chi}$. Then $\operatorname{deg} \omega_{i}=\operatorname{deg} Q_{\chi}-1$ for some $i$.

Proof: Since the 1 -form $d Q_{\chi}$ is $\chi$-invariant, $d Q_{\chi}=\sum_{i} h_{i} \omega_{i}$ for some homogeneous polynomials $h_{i}$ in $S^{G}$. Suppose none of the $h_{i}$ lie in $\mathbb{C}^{*}$. Fix a basis $x_{1}, \ldots, x_{\ell}$ of $V^{*}$. Then each $\partial / \partial x_{j}\left(Q_{\chi}\right)$ lies in $I$. By Euler's formula, $\left(\operatorname{deg} Q_{\chi}\right) Q_{\chi}=\left(\operatorname{deg} Q_{\chi}\right) \sum_{i} x_{i} \frac{\partial}{\partial x_{i}}\left(Q_{\chi}\right)$ also lies in $I$, contradicting Lemma 5. Hence, some $h_{i}$ is a nonzero scalar, and thus $\left\{\omega_{1}, \ldots, \omega_{i-1}, d Q_{\chi}, \omega_{i+1}, \ldots, \omega_{\ell}\right\}$ also generates $\Omega^{\chi}$.

We say that the character $\chi$ is wholly non-trivial (borrowing terminology from Victor Reiner) when $\chi\left(s_{H}\right) \neq 1$ for each $H$ in $\mathcal{A}$. Thus $\chi$ is wholly non-trivial exactly when $Q$ divides $Q_{\chi}$. Stanley's formula (Equation 2) for $Q_{\chi}$ directly implies

Lemma 11 Let $\chi$ be a linear character of $G$. Then $\chi$ is wholly nontrivial if and only if (up to a nonzero scalar)

$$
Q_{\chi \operatorname{det}} Q_{\bar{\chi} \operatorname{det}}=Q_{\operatorname{det}^{2}} .
$$

Proposition 12 Let $\chi$ be a linear character of $G$. Then $\chi$ is wholly nontrivial if and only if generators of $\Omega^{\chi}$ have degrees $\operatorname{deg} Q_{\chi}-m_{i}^{*}$ for $i=1, \ldots, \ell$. Furthermore, $\chi$ is trivial if and only if generators of $\Omega^{\chi}$ have degrees $\operatorname{deg} Q_{\chi}+m_{i}$ for $i=1, \ldots, \ell$.

Proof: Recall that generators of $\Omega^{\text {det }}$ have degrees $\operatorname{deg} Q_{\text {det }}-m_{i}^{*}$ for $i=1, \ldots, \ell$ (Corollary 8). Let $\omega_{1}, \ldots, \omega_{\ell}$ generate $\Omega^{\chi}$ with $\operatorname{deg} \omega_{i} \leq \operatorname{deg} \omega_{i+1}$. Then

$$
\omega_{1} \wedge \cdots \wedge \omega_{\ell}=Q_{\chi}^{\ell-1} Q_{\chi \operatorname{det}} \text { vol }
$$


by Theorem 2 , and hence

$$
Q_{\bar{\chi} \operatorname{det}} \omega_{1} \wedge \cdots \wedge Q_{\bar{\chi} \operatorname{det}} \omega_{\ell}=Q_{\bar{\chi} \text { det }} Q_{\operatorname{det}}^{\ell-1} Q_{\chi \operatorname{det}} \text { vol }
$$

by Lemma 6 . On the other hand, the $S^{G}$-module of det-invariant $\ell$-forms is generated by $Q_{\operatorname{det}^{2}}$ vol, and thus

$$
Q_{\bar{\chi} \operatorname{det}} \omega_{1} \wedge \cdots \wedge Q_{\bar{\chi} \text { det }} \omega_{\ell}=f Q_{\operatorname{det}^{\ell-1}} Q_{\operatorname{det}^{2}} \text { vol }
$$

for some $f$ in $S^{G}$ (see Equation 1). Hence, $Q_{\bar{\chi} \operatorname{det}} Q_{\chi \operatorname{det}}=f Q_{\operatorname{det}^{2}}$. But $\chi$ is wholly nontrivial exactly when $f$ is a nonzero constant (by Lemma 11), exactly when the $Q_{\bar{\chi}} \operatorname{det} \omega_{i}$ generate $\Omega^{\operatorname{det}}$ (by Theorem 2), exactly when the degree of each $Q_{\bar{\chi} \operatorname{det}} \omega_{i}$ is $\operatorname{deg} Q_{\operatorname{det}}-m_{i}^{*}$, and thus exactly when the degree of each $\omega_{i}$ is $\operatorname{deg} Q_{\chi}-m_{i}^{*}$ (by Lemma 6). Also note that if each $\operatorname{deg} \omega_{i}=\operatorname{deg} Q_{\chi}+m_{i}$, then

$$
\begin{aligned}
\operatorname{deg} Q_{\chi}^{\ell-1}+\operatorname{deg} Q_{\chi \operatorname{det}} & =\operatorname{deg} \omega_{1} \wedge \cdots \wedge \omega_{\ell}=\operatorname{deg} Q_{\chi}^{\ell}+m_{1}+\ldots+m_{\ell} \\
& =\operatorname{deg} Q_{\chi}^{\ell}+\operatorname{deg} Q_{\operatorname{det}}
\end{aligned}
$$

and $\operatorname{deg} Q_{\chi \operatorname{det}}=\operatorname{deg} Q_{\chi}+\operatorname{deg} Q_{\operatorname{det}}$. But Stanley's formula for $Q_{\chi}$ (Equation 2) implies that $\operatorname{deg} Q_{\chi \operatorname{det}}<\operatorname{deg} Q_{\chi}+\operatorname{deg} Q_{\text {det }}$ unless $\chi$ is trivial. Conversely, if $\chi$ is trivial, then $Q_{\chi}=1$ and we may take $\omega_{i}:=d f_{i}$.

We obtain some combinatorial identities by applying Lemmas 5 and 6 and Corollary 3 to Theorem 2, Propositions 9, 10, and 12, Lemma 7, and Corollary 4. Note that the coexponents are $m_{i}^{*}=e_{\ell-i}\left(V^{*}\right)$ in the corollary below, and recall that $e(\mathrm{det})$ is the number of reflections in $G$.

Corollary 13 Assume $G$ is irreducible. For any irreducible $G$-module $M$, label the $M$-exponents in increasing order: $e_{1}(M) \leq \cdots \leq e_{\operatorname{dim} M}(M)$. Let $\chi$ be any linear character of $G$. Then:

(a) $e(\chi \operatorname{det})=e(\operatorname{det})-e(\bar{\chi})$.

(b) $e_{1}(\chi V)+\cdots+e_{\ell}(\chi V)=(\ell-1) e(\chi)+e(\chi \operatorname{det})$.

(c) $e(\chi)-e_{\ell-i}\left(V^{*}\right) \leq e_{i}(\chi V) \leq e(\chi)+e_{i}(V)$ for $i=1, \ldots, \ell$.

(d) if $\chi \neq 1$, then some $e_{i}(\chi V)=e(\chi)-1$.

(e) $\chi$ is wholly nontrivial if and only if $e_{i}(\chi V)=e(\chi)-e_{\ell-i}\left(V^{*}\right)$ for $i=1, \ldots, \ell$.

(f) $\chi$ is trivial if and only if $e_{i}(\chi V)=e(\chi)+e_{i}(V)$ for $i=1, \ldots, \ell$. 
(g) $e_{i}(\bar{\chi} V)=e(\bar{\chi})-e(\chi)+e_{i}(\chi V)$ for $i=1, \ldots, \ell$.

(h) $e_{i}\left(\chi V^{*}\right)=e(\operatorname{det})-e_{i}(\bar{\chi} \operatorname{det} V)$ for $i=1, \ldots, \ell$.

\section{Springer's theory of regular elements}

The invariant theory of reflection groups generated by $\ell=\operatorname{dim} V$ reflections is particularly appealing. We recall Springer's theory of regular elements. A vector $v$ in $V$ is regular if its isotropy group in $G$ is trivial. Steinberg [21, Theorem 1.5] shows that $v$ is regular if and only if $v$ does not lie on any of the reflecting hyperplanes for $G$. When $g$ in $G$ has a regular eigenvector, then $g$ is a regular element and the order of $g$ is a regular number for $G$. Springer [18, Prop. 4.5] shows

Theorem 14 Let $g$ be a regular element of $G$ with order $d$. Let $\xi=e^{\frac{2 \pi i}{d}}$. Let $M$ be any irreducible representation of $G$. Then the eigenvalues of the action of $g$ on $M$ are $\xi^{-e_{1}}, \ldots, \xi^{-e_{\operatorname{deg} M}}$ where $e_{1}, \ldots, e_{\operatorname{deg} M}$ are the $M$-exponents.

Corollary 15 Let $G$ be an irreducible reflection group and let $\chi$ be a linear character of $G$. Let $d$ be a regular number for $G$. The exponents of the twisted reflection representation $\chi V$ are $\operatorname{deg} Q_{\chi}+m_{1}, \operatorname{deg} Q_{\chi}+m_{2}, \ldots, \operatorname{deg} Q_{\chi}+m_{\ell}$ modulo $d$.

Proof: Suppose $\xi=e^{\frac{2 \pi i}{d}}$ where $d$ is the order of a regular element $g$. By Lemma 5, $e(\chi)=\operatorname{deg} Q_{\chi}$. Apply Theorem 14 to $M=\mathbb{C}_{\chi}, M=V$, and $M=\chi V: \chi(g)=\xi^{-\operatorname{deg} Q_{\chi}}$ and the eigenvalues of $g$ on $V$ are $\xi^{m_{i}}$; hence the eigenvalues of $g$ on $\chi V$ are $\chi(g) \xi^{m_{i}}=$ $\xi^{-m_{i}-\operatorname{deg} Q_{\chi}}$ for $i=1, \ldots, \ell$.

If if $d$ is regular, then by Corollary 15 , there is a permutation $\pi$ of $1, \ldots, \ell$ such that the exponents and coexponents of $G$ satisfy $m_{i}+m_{\pi(i)}^{*} \equiv 0$ modulo $d$ (also see [8, Cor. 4.6]). Set $d_{\ell}:=m_{\ell}+1$. The group $G$ is a duality group if $d_{\ell}=m_{i}+m_{i}^{*}$ for each $i$. Examples include Coxeter groups and Shephard groups. Theorem 16 below implies that if $G$ is a duality group, then $d_{\ell}$ is a regular number. The converse is false, e.g., $d_{\ell}$ is regular for the group $G_{31}$, but $G_{31}$ is not a duality group.

Orlik and Solomon [11, Theorem 5.5] observe (among other equivalences) that $G$ is a duality group if and only if $G$ can be generated by $\ell=\operatorname{dim} V$ reflections. They examine the irreducible groups case-by-case. Bessis [2] gives a proof of this result which avoids case-by-case analysis using an observation by Lehrer and Springer [8]. Lehrer and Michel [7] give a case-free proof of this observation, which is the next theorem. The degrees of $G$ are the degrees of the basic invariants $m_{i}+1$ for $i=1, \ldots \ell$. The codegrees of $G$ are the integers $m_{i}^{*}-1$ for $i=1, \ldots \ell$.

Theorem 16 An integer $d$ is a regular number for $G$ if and only if $d$ divides as many degrees as codegrees. 
The following result is false for many non-duality groups.

Corollary 17 Let $G$ be a duality group and let $\chi$ be a linear character of $G$. Let $e_{1}(\chi V) \leq$ $\cdots \leq e_{\ell}(\chi V)$ be the $\chi V$-exponents. Then each $e_{i}(\chi V)$ is $e(\chi)+m_{i}$ or $e(\chi)-m_{i}^{*}$ for $i=1, \ldots, \ell$.

Proof: Since $G$ is a duality group, $d_{\ell}$ is a regular number by Theorem 16 and $e_{i}(\chi V) \equiv$ $\operatorname{deg} Q_{\chi}+m_{i} \equiv \operatorname{deg} Q_{\chi}-m_{i}^{*}$ modulo $d_{\ell}$ by Corollary 15 . The result then follows from Proposition 9 (see Corollary 13c).

\section{Constructing semi-invariant forms}

We show how to construct generators for semi-invariant forms using differential operators. (This method produces an explicit $\mathbb{C}$-basis for the isotypic component of the coinvariant algebra whose type is any twisted reflection representation.) We list the explicit $\chi V$-exponents and $\chi$-invariant forms for the irreducible reflection groups (except the infinite family) in tables at the end.

We may assume that the reflection group $G$ preserves a Hermitian inner product, $V \times V \rightarrow$ $\mathbb{C}$. The inner product induces a natural map from $S(V)$ to $S=S\left(V^{*}\right)$, say $p \mapsto \partial p$. Identify $S(V)$ with the algebra of differential operators to obtain a map

$$
\begin{aligned}
& S \times S \longrightarrow S \\
& (p, f) \mapsto(\partial p) f
\end{aligned}
$$

(where $(\partial p) f$ is the result of applying the differential operator $\partial p$ to $f$ ). This map preserves the group action: $(g \partial p)(g f)=g(\partial p(f))$ for every $g$ in $G$ and polynomials $p, f$ in $S$. This implies that the induced "star and bar" map from the product space of derivations and polynomials to the space of differential forms preserves semi-invariance:

Proposition 18 Let $\chi$ and $\tau$ be linear characters of $G$. The natural map

$$
\begin{aligned}
& (S \otimes V) \times S & \longrightarrow\left(S \otimes V^{*}\right) \\
\text { given by } & (p \otimes v, f) & \mapsto(\partial p) f \otimes \partial v \\
\text { induces a map } & (S \otimes V)^{\tau} \times S^{\chi} & \longrightarrow\left(S \otimes V^{*}\right)^{\chi \bar{\tau}} .
\end{aligned}
$$

Denote the image of a derivation $\theta$ and a polynomial $f \in S$ under this map by $\tilde{\theta} f$ (a differential form). Let $\widetilde{\omega} f$ (a derivation) denote the image of a differential form $\omega$ and a polynomial $f \in S$ under the analogous map $\left(S \otimes V^{*}\right)^{\tau} \times S^{\chi} \longrightarrow(S \otimes V)^{\chi \bar{\tau}}$. 
Corollary 19 If $f_{i}$ and $f_{j}$ are basic invariants, then $\widetilde{d f}_{i} f_{j}$ is an invariant derivation. If $\theta$ is a basic derivation, then $\widetilde{\theta} Q_{\chi}$ is a $\chi$-invariant 1-form.

We use the above corollary to construct basic derivations and generators for $\Omega^{x}$. These techniques are suggested by the numerology of Corollaries 13 and 17. Shephard and Todd [13] classify the irreducible reflection groups into an infinite family $G(r, p, \ell)$ and thirty-three exceptional groups labeled $G_{4}$ through $G_{37}$. Let $\theta_{1}, \ldots, \theta_{\ell}$ be a set of basic derivations with $\operatorname{deg} \theta_{i} \geq \operatorname{deg} \theta_{i+1}$. Let $f_{1}, \ldots, f_{\ell}$ be a set of basic invariants with $\operatorname{deg} f_{i} \leq \operatorname{deg} f_{i+1}$. When $G$ is a duality group, $\operatorname{deg}\left(\widetilde{d f_{i}} f_{\ell}\right)=\operatorname{deg} f_{\ell}-\operatorname{deg} d f_{i}=\operatorname{deg} \theta_{i}$. Hence, $\widetilde{d f}_{1} f_{\ell}, \ldots, \widetilde{d f}_{\ell} f_{\ell}$ form a set of invariant derivations with the same (polynomial) degrees as $\theta_{1}, \ldots, \theta_{\ell}$. Do they form a set of basic derivations? Similarly, does the set of $\chi$-invariant forms $\left\{\widetilde{\theta}_{1} Q_{\chi}, \ldots, \widetilde{\theta}_{\ell} Q_{\chi}, Q_{\chi} d f_{1}, \ldots, Q_{\chi} d f_{\ell}\right\}$ include generators of $\Omega^{\chi}$ ? Corollary 17 suggests that a generating set of $\Omega^{\chi}$ may be chosen from this set when $G$ is a duality group. We verify this suggestion in the observation below using basic invariants from Shephard and Todd [13]. The observation after suggests a pattern for nonduality groups as well. Both observations seem likely for the family $G(r, p, \ell)$ although we have not checked details.

Observation 20 Let $G$ be an irreducible duality group, $G \neq G(r, p, \ell)$. The basic invariants, $f_{1}, \ldots, f_{\ell}$, may be chosen so that $\left\{\widetilde{d f}_{1} f_{\ell}, \ldots, \widetilde{d f}_{\ell} f_{\ell}\right\}$ is a set of basic derivations. Let $\theta_{i}:=\widetilde{d f}_{i} f_{\ell}$ and let $\chi$ be a linear character of $G$. A generating set of $\Omega^{\chi}$ may be chosen from $\left\{\widetilde{\theta}_{1} Q_{\chi}, \ldots, \widetilde{\theta}_{\ell} Q_{\chi}, Q_{\chi} d f_{1}, \ldots, Q_{\chi} d f_{\ell}\right\}$.

Observation 21 Let $G$ be an irreducible reflection group, $G \neq G(r, p, \ell)$, and let $\chi$ be a linear character of $G$. There are basic invariants $f_{i}$ and invariant polynomials $F_{i}$ so that $\left\{\widetilde{d f}_{1} F_{1}, \ldots, \widetilde{d f}_{\ell} F_{\ell}\right\}$ is a set of basic derivations.

We give the explicit $\chi$ and $\chi V$-exponents and some illustrative examples in tables below. Klein's invariants [6] appear in Table 1. Table 2 gives basic derivations in terms of differential operators for the exceptional groups. (The Coxeter groups are omitted since the coefficients of each $\theta_{i}$ are just the coefficients of $d f_{\ell-i}$.) Table 3 list the exceptional groups and give the polynomial $Q_{\chi}$, its degree (the $\chi$-exponent $e(\chi)$ ), and generators of $\Omega^{\chi}$ and their degrees (the $\chi V$-exponents) for each linear character $\chi$ of $G$. We omit those duality groups whose only linear characters are $\overline{\mathrm{det}}$ and the trivial character, since these two cases are well understood. The symbol $\triangleright$ indicates a nonduality group throughout. The $\chi V$-exponents were first computed from character tables using a version of Molien's theorem and the software GAP and Mathematica. It may be interesting to note that for a fixed two-dimensional exceptional group, one may compute all the semi-invariant forms and derivations from just one polynomial. 
Table 1. Klein's invariants for 2-dim. groups.

$$
\begin{aligned}
& \Phi=x_{1}^{4}+2 i \sqrt{3} x_{1}^{2} x_{2}^{2}+x_{2}^{4} \\
& \Psi=x_{1}^{4}-2 i \sqrt{3} x_{1}^{2} x_{2}^{2}+x_{2}^{4} \\
& t=x_{1} x_{2}\left(x_{1}^{4}-x_{2}^{4}\right) \\
& W=x_{1}^{8}+14 x_{1}^{4} x_{2}^{4}+x_{2}^{8} \\
& X=x_{1}^{12}-33 x_{1}^{8} x_{2}^{4}-33 x_{1}^{4} x_{2}^{8}+x_{2}^{12} \\
& f=x_{1} x_{2}\left(x_{1}^{10}+11 x_{1}^{5} x_{2}^{5}-x_{2}^{10}\right) \\
& H=x_{1}^{20}-228 x_{1}^{15} x_{2}^{5}+494 x_{2}^{10} x_{2}^{10}+228 x_{1}^{5} x_{2}^{15}+x_{2}^{20} \\
& T=x_{1}^{30}+522 x_{1}^{25} x_{2}^{5}-10005 x_{1}^{20} x_{2}^{10}-10005 x_{1}^{10} x_{2}^{20}-522 x_{1}^{5} x_{2}^{25}+x_{2}^{30}
\end{aligned}
$$

\begin{tabular}{|c|c|c|c|c|}
\hline \multirow[b]{2}{*}{ Group } & \multicolumn{2}{|c|}{ Basic inv. } & \multicolumn{2}{|c|}{ Basic der. } \\
\hline & $f_{1}$ & $f_{2}$ & $\theta_{1}$ & $\theta_{2}$ \\
\hline 4 & $\Phi$ & $t$ & $\widetilde{d f_{1}} f_{2}$ & $\widetilde{d f}_{2} f_{2}$ \\
\hline 5 & $t$ & $\Phi^{3}$ & $\widetilde{d f_{1}} f_{2}$ & $\widetilde{d f}_{2} f_{2}$ \\
\hline 6 & $t^{2}$ & $\Phi$ & $\widetilde{d f_{1}} f_{2}$ & $\widetilde{d f}_{2} f_{2}$ \\
\hline-7 & $\Phi^{3}$ & $t^{2}$ & $\widetilde{d f_{1}} f_{2}^{2}$ & $\widetilde{d f}_{2} f_{2}$ \\
\hline 8 & $W$ & $X$ & $\widetilde{d f_{1}} f_{2}$ & $\widetilde{d f}_{2} f_{2}$ \\
\hline 9 & $W$ & $X^{2}$ & $\widetilde{d f}_{1} f_{2}$ & $\widetilde{d f}_{2} f_{2}$ \\
\hline 10 & $x$ & $W^{3}$ & $\widetilde{d f_{1}} f_{2}$ & $\widetilde{d f}_{2} f_{2}$ \\
\hline$\triangleright 11$ & $W^{3}$ & $X^{2}$ & $\widetilde{d f}_{1} f_{2}^{2}$ & $\widetilde{d f}_{2} f_{2}$ \\
\hline$\checkmark 12$ & $t$ & $W$ & $\widetilde{d f_{1}} f_{2}^{2}$ & $\widetilde{d f}_{2} f_{2}$ \\
\hline$\neg 13$ & $W$ & $t^{2}$ & $\widetilde{d f}_{1} f_{2}^{2}$ & $\widetilde{d f}_{2} f_{2}$ \\
\hline 14 & $t$ & $X^{2}$ & $\widetilde{d f_{1}} f_{2}$ & $\widetilde{d f}_{2} f_{2}$ \\
\hline$\checkmark 15$ & $t^{2}$ & $X^{2}$ & $\widetilde{d f}_{1}\left(f_{1} f_{2}\right)$ & $\widetilde{d f}_{2} f_{2}$ \\
\hline 16 & $H$ & $T$ & $\widetilde{d f_{1}} f_{2}$ & $\widetilde{d f}_{2} f_{2}$ \\
\hline 17 & $H$ & $T^{2}$ & $\widetilde{d f}_{1} f_{2}$ & $\widetilde{d f}_{2} f_{2}$ \\
\hline 18 & $T$ & $H^{3}$ & $\widetilde{d f_{1}} f_{2}$ & $\widetilde{d f}_{2} f_{2}$ \\
\hline$\rightarrow 19$ & $H^{3}$ & $T^{2}$ & $\widetilde{d f_{1}} f_{2}^{2}$ & $\widetilde{d f}_{2} f_{2}$ \\
\hline 20 & $f$ & $T$ & $\widetilde{d f_{1}} f_{2}$ & $\widetilde{d f}_{2} f_{2}$ \\
\hline 21 & $f$ & $T^{2}$ & $\widetilde{d f_{1}} f_{2}$ & $\widetilde{d f}_{2} f_{2}$ \\
\hline-22 & $f$ & $H$ & $\widetilde{d f_{1}} f_{2}^{2}$ & $\widetilde{d f}_{2} f_{2}$ \\
\hline Group & & $\mathrm{Bas}$ & er. $\theta_{i}$ & \\
\hline $24-27,29,32-24$ & $\widetilde{d f_{1}} f_{\ell}$ & $\widetilde{d f}_{2} f_{\ell}$ & & $\widetilde{d f}_{\ell} f_{\ell}$ \\
\hline - 31 & $\widetilde{d f}_{3} f_{4}^{2}$ & $\widetilde{d f}_{1} f_{4}$ & $\widetilde{d f}_{2} f_{4}$ & $\widetilde{d f}_{4} f_{4}$ \\
\hline
\end{tabular}

Table 2. Basic invariants and basic derivations. 
Table 3. Semi-invariants and $\chi V$-exponents.

\begin{tabular}{|c|c|c|c|c|c|}
\hline \multirow{2}{*}{$\frac{\text { Group }}{\mathrm{G}_{4}}$} & \multirow{2}{*}{$\frac{Q_{\chi}}{\Psi^{2}}$} & \multirow{2}{*}{$\frac{\text { Deg }}{8}$} & \multicolumn{2}{|c|}{ Generators of $\Omega^{x}$} & \multirow{2}{*}{$\frac{\chi V \operatorname{Exp}}{5,7}$} \\
\hline & & & $\widetilde{\theta_{1}} Q_{\chi}$ & $\widetilde{\theta_{2}} Q_{\chi}$ & \\
\hline & $\Psi$ & 4 & $\widetilde{\theta_{1}} Q_{\chi}$ & $\widetilde{\theta_{2}} Q_{\chi}$ & 1,3 \\
\hline \multirow[t]{8}{*}{$\mathrm{G}_{5}$} & $\Phi \Psi^{2}$ & 12 & $\tilde{\theta_{1}} Q_{\chi}$ & $\widetilde{\theta_{2}} Q_{\chi}$ & 5,11 \\
\hline & $\Phi^{2} \Psi$ & 12 & $\widetilde{\theta_{1}} Q_{\chi}$ & $\tilde{\theta}_{2} Q_{\chi}$ & 5,11 \\
\hline & $\Phi$ & 4 & $\tilde{\theta}_{2} Q_{\chi}$ & $d f_{1} Q_{\chi}$ & 3,9 \\
\hline & $\Phi^{2} \Psi^{2}$ & 16 & $\widetilde{\theta_{1}} Q_{\chi}$ & $\widetilde{\theta_{2}} Q_{\chi}$ & 9,15 \\
\hline & $\Psi$ & 4 & $\widetilde{\theta_{2}} Q_{\chi}$ & $d f_{1} Q_{\chi}$ & 3,9 \\
\hline & $\Psi^{2}$ & 8 & $\tilde{\theta_{2}} Q_{\chi}$ & $d f_{1} Q_{\chi}$ & 7,13 \\
\hline & $\Phi^{2}$ & 8 & $\widetilde{\theta_{2}} Q_{\chi}$ & $d f_{1} Q_{\chi}$ & 7,13 \\
\hline & $\Phi \Psi$ & 8 & $\widetilde{\theta_{1}} Q_{\chi}$ & $\widetilde{\theta_{2}} Q_{\chi}$ & 1,7 \\
\hline \multirow[t]{5}{*}{$\mathrm{G}_{6}$} & $t$ & 6 & $\widetilde{\theta_{2}} Q_{\chi}$ & $d f_{1} Q_{\chi}$ & 5,9 \\
\hline & $\Psi t$ & 10 & $\widetilde{\theta_{1}} Q_{\chi}$ & $\underset{\sim}{\tilde{\theta_{2}}} Q_{\chi}$ & 1,9 \\
\hline & $\Psi^{2} t$ & 14 & $\tilde{\theta}_{1} Q_{\chi}$ & $\tilde{\theta}_{2} Q_{x}$ & 5,13 \\
\hline & $\Psi$ & 4 & $\widetilde{\theta_{2}} Q_{\chi}$ & $d f_{1} Q_{\chi}$ & 3,7 \\
\hline & $\Psi^{2}$ & 8 & $\widetilde{\theta_{2}} Q_{\chi}$ & $d f_{1} Q_{\chi}$ & 7,11 \\
\hline \multirow[t]{17}{*}{$\triangleright \mathrm{G}_{7}$} & $t$ & 6 & $\widetilde{\theta_{2}} Q_{x}$ & $d f_{1} Q_{\chi}$ & 5,17 \\
\hline & $\Phi^{2} \Psi t$ & 18 & $\widetilde{\theta_{1}} Q_{\chi}$ & $\widetilde{\theta_{2}} Q_{\chi}$ & 5,17 \\
\hline & $\Phi \Psi^{2} t$ & 18 & $\widetilde{\theta_{1}} Q_{\chi}$ & $\widetilde{\theta_{2}} Q_{x}$ & 5,17 \\
\hline & $\Phi^{2} \Psi$ & 12 & $\tilde{\theta_{2}} Q_{\chi}$ & $\tilde{\theta_{1}}\left(f_{2} Q_{\chi}\right)$ & 11,11 \\
\hline & $\Phi \Psi^{2}$ & 12 & $\widetilde{\theta_{2}} Q_{\chi}$ & $\tilde{\theta_{1}}\left(f_{2} Q_{\chi}\right)$ & 11,11 \\
\hline & $\Phi^{2} \Psi^{2} t$ & 22 & $\tilde{\theta_{1}} Q_{\chi}$ & $\tilde{\theta_{2}} Q_{\chi}$ & 9,21 \\
\hline & $\Phi t$ & 10 & $\tilde{\theta}_{2} Q_{\chi}$ & $\widetilde{\theta_{1}}\left(f_{2} Q_{\chi}\right)$ & 9,9 \\
\hline & $\Psi t$ & 10 & $\widetilde{\theta_{2}} Q_{\chi}$ & $\tilde{\theta}_{1}\left(f_{2} Q_{\chi}\right)$ & 9,9 \\
\hline & $\Phi^{2} \Psi^{2}$ & 16 & $\tilde{\theta}_{2} Q_{x}$ & $\widetilde{\theta_{1}}\left(f_{2} Q_{\chi}\right)$ & 15,15 \\
\hline & $\Phi$ & 4 & $\widetilde{\theta_{2}} Q_{\chi}$ & $d f_{2} Q_{\chi}$ & 3,15 \\
\hline & $\Psi$ & 4 & $\widetilde{\theta_{2}} Q_{\chi}$ & $d f_{2} Q_{\chi}$ & 3,15 \\
\hline & $\Phi \Psi t$ & 14 & $\widetilde{\theta_{1}} Q_{\chi}$ & $\widetilde{\theta_{2}} Q_{\chi}$ & 1,13 \\
\hline & $\Psi^{2} t$ & 14 & $\widetilde{\theta_{2}} Q_{\chi}$ & $\tilde{\theta}_{1}\left(f_{2} Q_{\chi}\right)$ & 13,13 \\
\hline & $\Phi^{2} t$ & 14 & $\tilde{\theta}_{2} Q_{x}$ & $\widetilde{\theta_{1}}\left(f_{2} Q_{\chi}\right)$ & 13,13 \\
\hline & $\Phi \Psi$ & 8 & $\widetilde{\theta_{2}} Q_{\chi}$ & $\tilde{\theta_{1}}\left(f_{2} Q_{\chi}\right)$ & 7,7 \\
\hline & $\Phi^{2}$ & 8 & $\widetilde{\theta_{2}} Q_{\chi}$ & $d f_{2} Q_{\chi}$ & 7,19 \\
\hline & $\Psi^{2}$ & 8 & $\widetilde{\theta_{2}} Q_{\chi}$ & $d f_{2} Q_{\chi}$ & 7,19 \\
\hline \multirow[t]{3}{*}{$\mathrm{G}_{8}$} & $t^{2}$ & 12 & $\widetilde{\theta_{1}} Q_{\chi}$ & $\widetilde{\theta_{2}} Q_{\chi}$ & 7,11 \\
\hline & $t$ & 6 & $\widetilde{\theta_{1}} Q_{\chi}$ & $\widetilde{\theta_{2}} Q_{\chi}$ & 1,5 \\
\hline & $t^{3}$ & 18 & $\widetilde{\theta_{1}} Q_{\chi}$ & $\tilde{\theta_{2}} Q_{\chi}$ & 13,17 \\
\hline \multirow[t]{2}{*}{$\mathrm{G}_{9}$} & $t^{2}$ & 12 & $\tilde{\theta_{2}} Q_{\chi}$ & $d f_{1} Q_{\chi}$ & 11,19 \\
\hline & $X$ & 12 & $\widetilde{\theta_{2}} Q_{\chi}$ & $d f_{1} Q_{\chi}$ & 11,19 \\
\hline
\end{tabular}


Table 3. (Continued)

\begin{tabular}{|c|c|c|c|c|c|}
\hline \multirow[t]{2}{*}{ Group } & \multirow{2}{*}{$\frac{Q_{\chi}}{t^{2} \mathrm{X}}$} & \multirow{2}{*}{$\frac{\text { Deg }}{24}$} & \multicolumn{2}{|c|}{ Generators of $\Omega^{x}$} & \multirow{2}{*}{$\frac{\chi V \operatorname{Exp}}{7,23}$} \\
\hline & & & $\tilde{\theta_{1}} Q_{\chi}$ & $\tilde{\theta}_{2} Q_{\chi}$ & \\
\hline & $t^{3} \mathrm{X}$ & 30 & $\tilde{\theta_{1}} Q_{\chi}$ & $\tilde{\theta}_{2} Q_{\chi}$ & 13,29 \\
\hline & $t$ & 6 & $\tilde{\theta}_{2} Q_{\chi}$ & $d f_{1} Q_{\chi}$ & 5,13 \\
\hline & $t^{3}$ & 18 & $\tilde{\theta_{2}} Q_{\chi}$ & $d f_{1} Q_{\chi}$ & 17,25 \\
\hline & $t X$ & 18 & $\widetilde{\theta_{1}} Q_{\chi}$ & $\tilde{\theta_{2}} Q_{\chi}$ & 1,17 \\
\hline \multirow[t]{11}{*}{$\mathrm{G}_{10}$} & $t^{2}$ & 12 & $\tilde{\theta}_{2} Q_{\chi}$ & $d f_{1} Q_{\chi}$ & 11,23 \\
\hline & $t^{3}$ & 18 & $\tilde{\theta_{2}} Q_{\chi}$ & $d f_{1} Q_{\chi}$ & 17,29 \\
\hline & $t$ & 6 & $\widetilde{\theta_{2}} Q_{\chi}$ & $d f_{1} Q_{\chi}$ & 5,17 \\
\hline & $t^{3} W$ & 26 & $\tilde{\theta_{1}} Q_{\chi}$ & $\tilde{\theta}_{2} Q_{\chi}$ & 13,25 \\
\hline & $t W$ & 14 & $\tilde{\theta_{1}} Q_{\chi}$ & $\tilde{\theta}_{2} Q_{\chi}$ & 1,13 \\
\hline & $t^{2} W$ & 20 & $\widetilde{\theta_{1}} Q_{\chi}$ & $\widetilde{\theta_{2}} Q_{\chi}$ & 7,19 \\
\hline & $W$ & 8 & $\tilde{\theta}_{2} Q_{\chi}$ & $d f_{1} Q_{\chi}$ & 7,19 \\
\hline & $t^{3} W^{2}$ & 34 & $\tilde{\theta_{1}} Q_{\chi}$ & $\tilde{\theta}_{2} Q_{\chi}$ & 21,33 \\
\hline & $t W^{2}$ & 22 & $\tilde{\theta}_{1} Q_{\chi}$ & $\tilde{\theta_{2}} Q_{\chi}$ & 9,21 \\
\hline & $t^{2} W^{2}$ & 28 & $\tilde{\theta_{1}} Q_{\chi}$ & $\tilde{\theta}_{2} Q_{\chi}$ & 15,27 \\
\hline & $W^{2}$ & 16 & $\tilde{\theta_{2}} Q_{\chi}$ & $d f_{1} Q_{\chi}$ & 15,27 \\
\hline \multirow[t]{23}{*}{$\triangleright \mathrm{G}_{11}$} & $X$ & 12 & $\tilde{\theta_{2}} Q_{\chi}$ & $d f_{1} Q_{\chi}$ & 11,35 \\
\hline & $t^{2}$ & 12 & $\tilde{\theta}_{2} Q_{x}$ & $d f_{2} Q_{\chi}$ & 11,35 \\
\hline & $t^{2} X$ & 24 & $\tilde{\theta_{2}} Q_{\chi}$ & $\widetilde{\theta_{1}}\left(f_{2} Q_{\chi}\right)$ & 23,23 \\
\hline & $t^{3} X$ & 30 & $\tilde{\theta}_{2} Q_{\chi}$ & $\tilde{\theta_{1}}\left(f_{2} Q_{\chi}\right)$ & 29,29 \\
\hline & $t$ & 6 & $\tilde{\theta_{2}} Q_{\chi}$ & $d f_{2} Q_{\chi}$ & 5,29 \\
\hline & $t^{3}$ & 18 & $\tilde{\theta}_{2} Q_{\chi}$ & $d f_{2} Q_{\chi}$ & 17,41 \\
\hline & $t X$ & 18 & $\tilde{\theta_{2}} Q_{\chi}$ & $\tilde{\theta_{1}}\left(f_{2} Q_{\chi}\right)$ & 17,17 \\
\hline & $t^{3} W X$ & 38 & $\tilde{\theta_{1}} Q_{x}$ & $\tilde{\theta_{2}} Q_{\chi}$ & 13,37 \\
\hline & $t W$ & 14 & $\tilde{\theta_{2}} Q_{\chi}$ & $\tilde{\theta_{1}}\left(f_{2} Q_{\chi}\right)$ & 13,13 \\
\hline & $t^{3} W$ & 26 & $\tilde{\theta_{2}} Q_{\chi}$ & $\tilde{\theta_{1}}\left(f_{2} Q_{\chi}\right)$ & 25,25 \\
\hline & $t W X$ & 26 & $\tilde{\theta_{1}} Q_{\chi}$ & $\tilde{\theta}^{\tilde{\theta}} Q_{\chi}$ & 1,25 \\
\hline & $t^{2} W$ & 20 & $\tilde{\theta}_{2} Q_{x}$ & $\tilde{\theta}_{1}\left(f_{2} Q_{\chi}\right)$ & 19,19 \\
\hline & $W X$ & 20 & $\widetilde{\theta_{2}} Q_{\chi}$ & $\widetilde{\theta_{1}}\left(f_{2} Q_{\chi}\right)$ & 19,19 \\
\hline & $t^{2} W X$ & 32 & $\tilde{\theta_{1}} Q_{\chi}$ & $\tilde{\theta}_{2} Q_{\chi}$ & 7,31 \\
\hline & $W$ & 8 & $\tilde{\theta_{2}} Q_{\chi}$ & $d f_{2} Q_{\chi}$ & 7,31 \\
\hline & $t^{3} W^{2} X$ & 46 & $\tilde{\theta_{1}} Q_{\chi}$ & $\tilde{\theta}_{2} Q_{\chi}$ & 21,45 \\
\hline & $t W^{2}$ & 22 & $\tilde{\theta}_{2} Q_{\chi}$ & $\tilde{\theta_{1}}\left(f_{2} Q_{\chi}\right)$ & 21,21 \\
\hline & $t^{3} W^{2}$ & 34 & $\tilde{\theta}_{2} Q_{x}$ & $\tilde{\theta_{1}}\left(f_{2} Q_{\chi}\right)$ & 33,33 \\
\hline & $t W^{2} X$ & 34 & $\tilde{\theta_{1}} Q_{\chi}$ & $\tilde{\theta_{2}} Q_{\chi}$ & 9,33 \\
\hline & $W^{2} X$ & 28 & $\tilde{\theta_{2}} Q_{\chi}$ & $\tilde{\theta_{1}}\left(f_{2} Q_{\chi}\right)$ & 27,27 \\
\hline & $t^{2} W^{2}$ & 28 & $\tilde{\theta}_{2} Q_{\chi}$ & $\tilde{\theta}_{1}\left(f_{2} Q_{\chi}\right)$ & 27,27 \\
\hline & $t^{2} W^{2} X$ & 40 & $\tilde{\theta_{1}} Q_{\chi}$ & $\tilde{\theta_{2}} Q_{\chi}$ & 15,39 \\
\hline & $W^{2}$ & 16 & $\widetilde{\theta_{2}} Q_{\chi}$ & $d f_{2} Q_{\chi}$ & 15,39 \\
\hline
\end{tabular}


Table 3. (Continued).

\begin{tabular}{|c|c|c|c|c|c|}
\hline \multirow{2}{*}{$\frac{\text { Group }}{\triangleright \mathrm{G}_{12}}$} & \multirow{2}{*}{$\frac{Q_{\chi}}{X}$} & \multirow{2}{*}{$\frac{\text { Deg }}{12}$} & \multicolumn{2}{|c|}{ Generators of $\Omega^{x}$} & \multirow{2}{*}{$\frac{\chi V \operatorname{Exp}}{1,11}$} \\
\hline & & & $\tilde{\theta_{1}} Q_{\chi}$ & $\tilde{\theta}_{2} Q_{\chi}$ & \\
\hline \multirow[t]{3}{*}{$\triangleright \mathrm{G}_{13}$} & $t X$ & 18 & $\tilde{\theta}_{1} Q_{\chi}$ & $\tilde{\theta}_{2} Q_{\chi}$ & 1,17 \\
\hline & $t$ & 6 & $\tilde{\theta}_{2} Q_{\chi}$ & $d f_{1} Q_{\chi}$ & 5,13 \\
\hline & $X$ & 12 & $\tilde{\theta}_{1}\left(f_{2} Q_{\chi}\right)$ & $\tilde{\theta}_{2} Q_{\chi}$ & 7,11 \\
\hline \multirow[t]{5}{*}{$\mathrm{G}_{14}$} & $X$ & 12 & $\tilde{\theta}_{2} Q_{\chi}$ & $d f_{1} Q_{\chi}$ & 11,17 \\
\hline & $W X$ & 20 & $\tilde{\theta_{1}} Q_{\chi}$ & $\tilde{\theta_{2}} Q_{\chi}$ & 1,19 \\
\hline & $W$ & 8 & $\tilde{\theta}_{2} Q_{\chi}$ & $d f_{1} Q_{\chi}$ & 7,13 \\
\hline & $W^{2} X$ & 28 & $\tilde{\theta_{1}} Q_{\chi}$ & $\tilde{\theta}_{2} Q_{\chi}$ & 9,27 \\
\hline & $W^{2}$ & 16 & $\tilde{\theta}_{2} Q_{\chi}$ & $d f_{1} Q_{\chi}$ & 15,21 \\
\hline \multirow[t]{11}{*}{$\triangleright \mathrm{G}_{15}$} & $t$ & 6 & $\tilde{\theta}_{2} Q_{\chi}$ & $d f_{2} Q_{\chi}$ & 5,29 \\
\hline & $t X$ & 18 & $\tilde{\theta}_{2} Q_{\chi}$ & $\tilde{\theta_{1}}\left(f_{2} Q_{\chi}\right)$ & 17,17 \\
\hline & $X$ & 12 & $\tilde{\theta}_{2} Q_{\chi}$ & $d f_{1} Q_{\chi}$ & 11,23 \\
\hline & $t W^{2}$ & 22 & $\tilde{\theta}_{2} Q_{\chi}$ & $\tilde{\theta_{1}}\left(f_{2} Q_{\chi}\right)$ & 21,21 \\
\hline & $t W^{2} X$ & 34 & $\tilde{\theta}_{1} Q_{\chi}$ & $\tilde{\theta}_{2} Q_{\chi}$ & 9,33 \\
\hline & $W^{2} X$ & 28 & $\tilde{\theta}_{1}\left(f_{1} Q_{\chi}\right)$ & $\tilde{\theta}_{2} Q_{\chi}$ & 15,27 \\
\hline & $W^{2}$ & 16 & $\tilde{\theta}_{2} Q_{\chi}$ & $d f_{1} Q_{\chi}$ & 15,27 \\
\hline & $t W$ & 14 & $\tilde{\theta}_{2} Q_{\chi}$ & $\tilde{\theta_{1}}\left(f_{2} Q_{\chi}\right)$ & 13,13 \\
\hline & $t W X$ & 26 & $\tilde{\theta}_{2} Q_{\chi}$ & $\tilde{\theta_{1}} Q_{\chi}$ & 1,25 \\
\hline & $W X$ & 20 & $\tilde{\theta}_{1}\left(f_{1} Q_{\chi}\right)$ & $\tilde{\theta}_{2} Q_{\chi}$ & 7,19 \\
\hline & $W$ & 8 & $\tilde{\theta}_{2} Q_{\chi}$ & $d f_{1} Q_{\chi}$ & 7,19 \\
\hline \multirow[t]{4}{*}{$\mathrm{G}_{16}$} & $f^{3}$ & 36 & $\tilde{\theta}_{1} Q_{\chi}$ & $\tilde{\theta}_{2} Q_{\chi}$ & 25,35 \\
\hline & $f$ & 12 & $\tilde{\theta_{1}} Q_{\chi}$ & $\tilde{\theta}_{2} Q_{\chi}$ & 1,11 \\
\hline & $f^{4}$ & 48 & $\tilde{\theta}_{1} Q_{\chi}$ & $\tilde{\theta}_{2} Q_{\chi}$ & 37,47 \\
\hline & $f^{2}$ & 24 & $\tilde{\theta_{1}} Q_{\chi}$ & $\tilde{\theta}_{2} Q_{\chi}$ & 13,23 \\
\hline \multirow[t]{9}{*}{$\mathrm{G}_{17}$} & $T$ & 30 & $\tilde{\theta}_{2} Q_{\chi}$ & $d f_{1} Q_{\chi}$ & 29,49 \\
\hline & $f^{4} T$ & 78 & $\tilde{\theta_{1}} Q_{\chi}$ & $\tilde{\theta}_{2} Q_{\chi}$ & 37,77 \\
\hline & $f^{3} T$ & 66 & $\tilde{\theta_{1}} Q_{\chi}$ & $\tilde{\theta}_{2} Q_{\chi}$ & 25,65 \\
\hline & $f^{2} T$ & 54 & $\tilde{\theta}_{1} Q_{\chi}$ & $\tilde{\theta}_{2} Q_{\chi}$ & 13,53 \\
\hline & $f T$ & 42 & $\tilde{\theta_{1}} Q_{\chi}$ & $\tilde{\theta}_{2} Q_{\chi}$ & 1,41 \\
\hline & $f^{4}$ & 48 & $\tilde{\theta}_{2} Q_{\chi}$ & $d f_{1} Q_{\chi}$ & 47,67 \\
\hline & $f^{3}$ & 36 & $\tilde{\theta}_{2} Q_{\chi}$ & $d f_{1} Q_{\chi}$ & 35,55 \\
\hline & $f^{2}$ & 24 & $\tilde{\theta}_{2} Q_{x}$ & $d f_{1} Q_{\chi}$ & 23,43 \\
\hline & $f$ & 12 & $\tilde{\theta}_{2} Q_{\chi}$ & $d f_{1} Q_{\chi}$ & 11,31 \\
\hline
\end{tabular}


Table 3. (Continued).

\begin{tabular}{|c|c|c|c|c|c|}
\hline \multirow{2}{*}{$\frac{\text { Group }}{\mathrm{G}_{18}}$} & \multirow{2}{*}{$\frac{Q_{\chi}}{f^{2}}$} & \multirow{2}{*}{$\frac{\text { Deg }}{24}$} & \multicolumn{2}{|c|}{ Generators of $\Omega^{x}$} & \multirow{2}{*}{$\frac{\chi V \operatorname{Exp}}{23,53}$} \\
\hline & & & $\widetilde{\theta_{2}} Q_{\chi}$ & $d f_{1} Q_{\chi}$ & \\
\hline & $f^{4}$ & 48 & $\widetilde{\theta_{2}} Q_{\chi}$ & $d f_{1} Q_{\chi}$ & 47,77 \\
\hline & $f$ & 12 & $\widetilde{\theta_{2}} Q_{\chi}$ & $d f_{1} Q_{\chi}$ & 11,41 \\
\hline & $f^{3}$ & 36 & $\widetilde{\theta_{2}} Q_{\chi}$ & $d f_{1} Q_{\chi}$ & 35,65 \\
\hline & $H$ & 20 & $\tilde{\theta_{2}} Q_{\chi}$ & $d f_{1} Q_{\chi}$ & 19,49 \\
\hline & $f^{2} H$ & 44 & $\tilde{\theta_{1}} Q_{\chi}$ & $\tilde{\theta}_{2} Q_{\chi}$ & 13,43 \\
\hline & $f^{4} H$ & 68 & $\widetilde{\theta_{1}} Q_{\chi}$ & $\tilde{\theta}_{2} Q_{\chi}$ & 37,67 \\
\hline & $f H$ & 32 & $\widetilde{\theta_{1}} Q_{\chi}$ & $\tilde{\theta}_{2} Q_{\chi}$ & 1,31 \\
\hline & $f^{3} H$ & 56 & $\tilde{\theta_{1}} Q_{\chi}$ & $\tilde{\theta}_{2} Q_{\chi}$ & 25,55 \\
\hline & $H^{2}$ & 40 & $\tilde{\theta_{2}} Q_{\chi}$ & $d f_{1} Q_{\chi}$ & 39,69 \\
\hline & $f^{2} H^{2}$ & 64 & $\tilde{\theta_{1}} Q_{\chi}$ & $\tilde{\theta}_{2} Q_{\chi}$ & 33,63 \\
\hline & $f^{4} H^{2}$ & 88 & $\widetilde{\theta_{1}} Q_{\chi}$ & $\tilde{\theta}_{2} Q_{\chi}$ & 57,87 \\
\hline & $f H^{2}$ & 52 & $\tilde{\theta_{1}} Q_{\chi}$ & $\tilde{\theta}_{2} Q_{\chi}$ & 21,51 \\
\hline & $f^{3} H^{2}$ & 76 & $\tilde{\theta_{1}} Q_{\chi}$ & $\tilde{\theta}_{2} Q_{\chi}$ & 45,75 \\
\hline \multirow[t]{22}{*}{$\triangleright \mathrm{G}_{19}$} & $T$ & 30 & $\tilde{\theta}_{2} Q_{\chi}$ & $d f_{1} Q_{\chi}$ & 29,89 \\
\hline & $f^{4} T$ & 78 & $\tilde{\theta_{2}} Q_{\chi}$ & $\tilde{\theta_{1}}\left(f_{2} Q_{\chi}\right)$ & 77,77 \\
\hline & $f^{3} T$ & 66 & $\widetilde{\theta_{2}} Q_{\chi}$ & $\tilde{\theta_{1}}\left(f_{2} Q_{\chi}\right)$ & 65,65 \\
\hline & $f^{2} T$ & 54 & $\tilde{\theta}_{2} Q_{\chi}$ & $\tilde{\theta_{1}}\left(f_{2} Q_{\chi}\right)$ & 53,53 \\
\hline & $f T$ & 42 & $\widetilde{\theta_{2}} Q_{\chi}$ & $\tilde{\theta_{1}}\left(f_{2} Q_{\chi}\right)$ & 41,41 \\
\hline & $f^{4}$ & 48 & $\widetilde{\theta_{2}} Q_{\chi}$ & $d f_{2} Q_{\chi}$ & 47,107 \\
\hline & $f^{3}$ & 36 & $\tilde{\theta}_{2} Q_{\chi}$ & $d f_{2} Q_{\chi}$ & 35,95 \\
\hline & $f^{2}$ & 24 & $\widetilde{\theta_{2}} Q_{\chi}$ & $d f_{2} Q_{\chi}$ & 23,83 \\
\hline & $f$ & 12 & $\tilde{\theta}_{2} Q_{\chi}$ & $d f_{2} Q_{\chi}$ & 11,71 \\
\hline & $H T$ & 50 & $\tilde{\theta}_{2} Q_{\chi}$ & $\widetilde{\theta_{1}}\left(f_{2} Q_{\chi}\right)$ & 49,49 \\
\hline & $f^{4} H T$ & 98 & $\tilde{\theta_{1}} Q_{\chi}$ & $\tilde{\theta}_{2} Q_{\chi}$ & 37,97 \\
\hline & $f^{3} H T$ & 86 & $\tilde{\theta_{1}} Q_{\chi}$ & $\tilde{\theta}_{2} Q_{\chi}$ & 25,85 \\
\hline & $f^{2} H T$ & 74 & $\tilde{\theta_{1}} Q_{\chi}$ & $\tilde{\theta}_{2} Q_{\chi}$ & 13,73 \\
\hline & $f H T$ & 62 & $\widetilde{\theta_{1}} Q_{\chi}$ & $\widetilde{\theta_{2}} Q_{\chi}$ & 1,61 \\
\hline & $H$ & 20 & $\widetilde{\theta_{2}} Q_{\chi}$ & $d f_{2} Q_{\chi}$ & 19,79 \\
\hline & $f^{4} H$ & 68 & $\tilde{\theta_{2}} Q_{\chi}$ & $\tilde{\theta_{1}}\left(f_{2} Q_{\chi}\right)$ & 67,67 \\
\hline & $f^{3} H$ & 56 & $\widetilde{\theta_{2}} Q_{\chi}$ & $\tilde{\theta_{1}}\left(f_{2} Q_{\chi}\right)$ & 55,55 \\
\hline & $f^{2} H$ & 44 & $\underset{\sim}{\tilde{\theta_{2}}} Q_{\chi}$ & $\underset{\sim}{\tilde{\theta_{1}}}\left(f_{2} Q_{\chi}\right)$ & 43,43 \\
\hline & $f H$ & 32 & $\tilde{\theta}_{2} Q_{\chi}$ & $\tilde{\theta_{1}}\left(f_{2} Q_{\chi}\right)$ & 31,31 \\
\hline & $H^{2} T$ & 70 & $\widetilde{\theta_{2}} Q_{\chi}$ & $\tilde{\theta_{1}}\left(f_{2} Q_{\chi}\right)$ & 69,69 \\
\hline & $f^{4} H^{2} T$ & 118 & $\widetilde{\theta_{1}} Q_{\chi}$ & $\tilde{\theta}_{2} Q_{\chi}$ & 57,117 \\
\hline & $f^{3} H^{2} T$ & 106 & $\tilde{\theta_{1}} Q_{\chi}$ & $\widetilde{\theta_{2}} Q_{\chi}$ & 45,105 \\
\hline
\end{tabular}


Table 3. (Continued)

\begin{tabular}{|c|c|c|c|c|c|c|}
\hline \multirow[t]{2}{*}{ Group } & \multirow{2}{*}{$\begin{array}{c}Q_{\chi} \\
f^{2} H^{2} T\end{array}$} & \multicolumn{2}{|r|}{ Deg } & \multicolumn{2}{|c|}{ Generators of $\Omega^{x}$} & \multirow{2}{*}{$\frac{\chi V \text { Exp }}{33,93}$} \\
\hline & & & 94 & $\tilde{\theta_{1}} Q_{\chi}$ & $\tilde{\theta_{2}} Q_{\chi}$ & \\
\hline & $f H^{2} T$ & & 82 & $\tilde{\theta_{1}} Q_{\chi}$ & $\tilde{\theta}_{2} Q_{\chi}$ & 21,81 \\
\hline & $H^{2}$ & & 40 & $\tilde{\theta_{2}} Q_{\chi}$ & $d f_{2} Q_{\chi}$ & 39,99 \\
\hline & $f^{4} H^{2}$ & & 88 & $\tilde{\theta}_{2} Q_{\chi}$ & $\tilde{\theta_{1}}\left(f_{2} Q_{\chi}\right)$ & 87,87 \\
\hline & $f^{3} H^{2}$ & & 76 & $\tilde{\theta_{2}} Q_{\chi}$ & $\tilde{\theta}_{1}\left(f_{2} Q_{\chi}\right)$ & 75,75 \\
\hline & $f^{2} H^{2}$ & & 64 & $\tilde{\theta}_{2} Q_{\chi}$ & $\tilde{\theta_{1}}\left(f_{2} Q_{\chi}\right)$ & 63,63 \\
\hline & $f H^{2}$ & & 52 & $\tilde{\theta_{2}} Q_{\chi}$ & $\tilde{\theta}_{1}\left(f_{2} Q_{\chi}\right)$ & 51,51 \\
\hline \multirow[t]{2}{*}{$\mathrm{G}_{20}$} & $H^{2}$ & & 40 & $\tilde{\theta_{1}} Q_{\chi}$ & $\tilde{\theta_{2}} Q_{\chi}$ & 21,39 \\
\hline & $H$ & & 20 & $\widetilde{\theta_{1}} Q_{\chi}$ & $\tilde{\theta}_{2} Q_{\chi}$ & 1,19 \\
\hline \multirow[t]{5}{*}{$\mathrm{G}_{21}$} & $\mathrm{~T}$ & & 30 & $\tilde{\theta}_{2} Q_{\chi}$ & $d f_{1} Q_{\chi}$ & 29,41 \\
\hline & $H^{2} \mathrm{~T}$ & & 70 & $\tilde{\theta_{1}} Q_{\chi}$ & $\widetilde{\theta_{2}} Q_{\chi}$ & 21,69 \\
\hline & $H^{2}$ & & 40 & $\widetilde{\theta_{2}} Q_{\chi}$ & $d f_{1} Q_{\chi}$ & 39,51 \\
\hline & $H T$ & & 50 & $\tilde{\theta_{1}} Q_{\chi}$ & $\tilde{\theta_{2}} Q_{\chi}$ & 1,49 \\
\hline & $H$ & & 20 & $\tilde{\theta}_{2} Q_{\chi}$ & $d f_{1} Q_{\chi}$ & 19,31 \\
\hline$\triangleright \mathrm{G}_{22}$ & $T$ & & 30 & $\tilde{\theta_{1}} Q_{\chi}$ & $\tilde{\theta_{2}} Q_{\chi}$ & 1,29 \\
\hline \multirow[t]{2}{*}{$\mathrm{G}_{25}$} & 12 & & $\tilde{\sigma}_{1} Q_{\chi}$ & $\widetilde{\theta_{2}} Q_{\chi}$ & $\tilde{\theta}_{3} Q_{\chi}$ & $5,8,11$ \\
\hline & 24 & & $\tilde{\theta}_{1} Q_{x}$ & $\widetilde{\theta_{2}} Q_{\chi}$ & $\widetilde{\theta_{3}} Q_{x}$ & $17,20,23$ \\
\hline \multirow[t]{5}{*}{$\mathrm{G}_{26}$} & 21 & & $\tilde{T}_{1} Q_{\chi}$ & $\tilde{\theta_{2}} Q_{\chi}$ & $\tilde{\theta_{3}} Q_{\chi}$ & $8,14,20$ \\
\hline & 33 & & $\tilde{\theta}_{1} Q_{\chi}$ & $\tilde{\theta}_{2} Q_{\chi}$ & $\tilde{\theta}_{3} Q_{\chi}$ & $20,26,32$ \\
\hline & 24 & & $\tilde{\theta}_{1} Q_{\chi}$ & $\tilde{\theta_{2}} Q_{\chi}$ & $d f_{1} Q_{\chi}$ & $17,23,29$ \\
\hline & 12 & & $\tilde{T}_{1} Q_{\chi}$ & $\tilde{\theta_{2}} Q_{\chi}$ & $d f_{1} Q_{\chi}$ & $5,11,17$ \\
\hline & 9 & & $\tilde{\theta}_{1} Q_{\chi}$ & $d f_{1} Q_{\chi}$ & $d f_{2} Q_{\chi}$ & $8,14,20$ \\
\hline \multirow[t]{4}{*}{ Group } & $\operatorname{deg} Q_{\chi}$ & Deg & \multicolumn{2}{|c|}{ Generators of $\Omega^{x}$} & $\chi^{V} \operatorname{Exp}$ & \\
\hline & 24 & $\tilde{\theta}_{1} Q_{\chi}$ & $\tilde{\theta_{2}} Q_{\chi}$ & $\tilde{\theta}_{3} Q_{\chi}$ & $\tilde{\theta_{4}} Q_{\chi}$ & $13,17,19,23$ \\
\hline & 12 & $\tilde{\theta}_{3} Q_{\chi}$ & $\tilde{\theta_{4}} Q_{\chi}$ & $d f_{1} Q_{\chi}$ & $d f_{2} Q_{\chi}$ & $7,11,13,17$ \\
\hline & 12 & $\tilde{\theta}_{3} Q_{\chi}$ & $\tilde{\theta}_{\sim} Q_{\chi}$ & $d f_{1} Q_{\chi}$ & $d f_{2} Q_{\chi}$ & $7,11,13,17$ \\
\hline$\triangleright \mathrm{G}_{31}$ & 60 & $\tilde{\theta}_{1} Q_{\chi}$ & $\tilde{\theta_{2}} Q_{\chi}$ & $\tilde{\theta}_{3} Q_{\chi}$ & $\tilde{\theta_{4}} Q_{\chi}$ & $31,43,47,59$ \\
\hline$G_{32}$ & 80 & $\widetilde{\theta_{1}} Q_{\chi}$ & $\tilde{\theta_{2}} Q_{\chi}$ & $\widetilde{\theta_{3}} Q_{\chi}$ & $\tilde{\theta_{4}} Q_{\chi}$ & $61,67,73,79$ \\
\hline & 40 & $\tilde{\theta}_{1} Q_{\chi}$ & $\tilde{\theta_{2}} Q_{\chi}$ & $\tilde{\theta}_{3} Q_{\chi}$ & $\widetilde{\theta_{4}} Q_{\chi}$ & $21,27,33,39$ \\
\hline
\end{tabular}

\section{Acknowledgments}

The author thanks Gus Lehrer for pointing out a shorter version of the proof of Proposition 1 and Hiroaki Terao for helpful discussions. 


\section{References}

1. S. Ariki, T. Terasoma, and H.-F. Yamada, "Higher Specht polynomials," Hiroshima Math. J. 27(1) (1997), 177-188.

2. D. Bessis, "Zariski theorems and diagrams for braid groups," Invent. Math., Invent. Math. 145(3) (2001), 487-507.

3. M. Broué, G. Malle, and J. Michel, “Towards Spetses I,” Transform. Groups 4(2-3) (1999), 157-218.

4. C. Chevalley, 'Invariants of finite groups generated by reflections," Amer. J. Math. 77 (1955), 778-782.

5. R. Kane, Reflection Groups and Invariant Theory, vol. 5 of CMS Books in Mathematics, Springer-Verlag, 2001.

6. F. Klein, Gesammelte Mathematische Abhandlungen. vol. 2, Springer, 1922.

7. G. Lehrer and J. Michel, "Invariant theory and eigenspaces for unitary reflection groups," submitted.

8. G. Lehrer and T. Springer, "Reflection subquotients of unitary reflection groups," Canad. J. Math. 51(6) (1999), 1175-1193.

9. H. Morita and H.-F. Yamada, "Higher Specht polynomials for the complex reflection groups $G(r, p, n)$," Hokkaido Math. J. 27(3) (1998), 505-515.

10. P. Orlik, V. Reiner, and A. Shepler, "The sign representation for Shephard groups," Mathematische Annalen 322 (2002), 477-492.

11. P. Orlik, and L. Solomon, "Unitary reflection groups and cohomology," Invent. Math. 59 (1980), 77-94.

12. P. Orlik and H. Terao, Arrangements of Hyperplanes, Springer-Verlag, Berlin, 1992.

13. G. Shephard and J. Todd, "Finite unitary reflection groups," Canad. J. Math. 6 (1954), 274-304.

14. A. Shepler, "Semi-invariants of finite reflection groups," J. Algebra 220 (1999), 314-326.

15. L. Smith, Polynomial Invariants of Finite Groups, vol. 6 of Research Notes in Mathematics. A K Peters, 1995.

16. L. Solomon, "Invariants of finite reflection groups," Nagoya Math. J. 22 (1963), 57-64.

17. L. Solomon, "Invariants of Euclidean reflection groups," Trans. Amer. Math. Soc. 113 (1964), $274-286$.

18. T. Springer, "Regular elements of finite reflection groups," Invent. Math. 25 (1974), 159-198.

19. R. Stanley, "Relative invariants of finite groups," Journal of Algebra 49(1) (1977), 134-148.

20. R. Steinberg, "Invariants of finite reflection groups," Canad. J. Math. 12 (1960), 616-618.

21. R. Steinberg, "Differential equations invariant under finite reflection groups," Trans. Amer. Math. Soc. 112 (1964), 392-400.

22. J. Stembridge, "On the eigenvalues of representations of reflection groups and wreath products," Pacific J. of Math. 140(2) (1989), 353-396.

23. H. Terao, "The Jacobians and the discriminants of finite reflection groups," Tohoku Math. J. 41 (1989), $237-247$. 